\title{
Skew-Type I Generalized Logistic Distribution and its Properties
}

\author{
Abdallah Mohamed Abdelfattah \\ Cairo University, Institute of statistical studies \& research, Egypt \\ a_afattah@hotmail.com
}

\begin{abstract}
Generalized logistic distributions are very versatile and give useful representations of many physical situations. Skew-symmetric densities recently received much attention in the literature. In this paper, we introduce a new class of skew-symmetric distributions which are formulated based on cumulative distributions of skew-symmetric densities. We derive, the probability density function (pdf) and cumulative distribution function (CDF) of the skew type I generalized logistic distribution denoted by $\operatorname{S'GLD}(\alpha, \beta, \lambda)$. The general statistical properties of the $\operatorname{S} \operatorname{GLD}(\alpha, \beta, \lambda)$ such as: the moment generating function (mgf), characteristic function (chf), Laplace and Fourier transformations are obtained in explicit form. Expressions for the $\mathrm{n}^{\text {th }}$ moment, skewness and kurtosis are discussed. Mean deviation about the mean and about the median, Renye entropy and the order statistics are also given. We consider the general case by inclusion of location and scale parameters. The results of Nadarajah (2009) are obtained as special cases. Graphical illustration of some results has been represented. Further we present a numerical example to illustrate some results of this paper.
\end{abstract}

Keywords: Skew type I generalized logistic distribution, Moment generating function, Skewness, Kurtosis, Mean deviation, Order statistics.

Mathematics Subject Classification 60E10, 62E10.

\section{1- Introduction}

The fundamental properties and characterization of symmetric distributions about origin are widely used in many applications as stated in Johnson et al. (1995). The logistic distribution has important uses in describing growth and as alternative for the normal distribution. It has several important applications in population modeling, biological, geological issues, psychological issues, actuarial, industrial and engineering felids. Different properties and applications of logistic distribution are studied extensively by, Ojo (1997, 2002, 2003), Olapade (2004), Alvarez et al. (2011), and Nassar and Elmasry (2012).

In the recent years, there has been quite an intense activity connected to a broad class of continuous probability distribution which are generated starting from a symmetric distribution and applying a suitable form perturbation of the symmetry. Univariate skewsymmetric models have been defined by several authors, using the concept described in the article by Azzalini (1985). As general result, Azzalini (1985) showed that any symmetric distribution was viewed as a member of more general class of skewed distribution. The skew-symmetric models defined based on the skew-normal distribution as

$f(x, \lambda)=2 h(x) . H(\lambda x),-\infty<x<\infty$

Where $\lambda \in \mathrm{R}$ is theskewness parameter, $\mathrm{h}(\mathrm{x})$ and $\mathrm{H}(\mathrm{x})$ are respectively the pdf and CDF of $N(0,1)$. 
In fact, when $\mathrm{h}($.$) and \mathrm{H}($.$) are the pdf and CDF of the standard logistic distribution$ respectively, then (1.1) is called skew-logistic distribution SLD with skewness parameter $\lambda$.The SLD $(\lambda)$ like other skew symmetric distribution can be used to model both positively and negatively skewed data due to one or more reasons, such as presence of outliers, small number of observations presence of other process, etc. The SLD and its statistical properties was first studied by Wahed and Ali (2001). They considered the pdf and CDF of standard logistic distribution to obtain SLD:



Hence, the pdf of $\operatorname{SLD}(\lambda)$ is

$$
f(x, \lambda)=\frac{2 e^{-x}}{\left(1+e^{-x}\right)^{2}\left(1+e^{-\lambda x}\right)}, \quad-\infty<x<\infty
$$

Nadarajah (2009) extended the SLD by introducing a scale parameter and studied its statistical properties. Gupta and Kundu (2010) used this distribution as generalized oflogistic distribution. They showed that the $\operatorname{SLD}(\lambda)$ is more flexible than the skew normal distribution for data analysis purposes. Many properties of SLD $(\lambda)$ and skew normal distribution are quite similar. The $\operatorname{SLD}(\lambda)$ and its statistical properties have been considered by many others, see, for example, Gupta et al.(2002), Olapade (2005), Nadarajah and Kotz (2006),(2007), Chakraborty el at.(2012), and Asgharzadeh et al. (2013).

Balakrishnan and leung (1988) defined the Type I generalized logistic distribution (Type I GLD) as one of three generalized forms of the standard logistic distribution. The twoparameter Type $\operatorname{I~} \operatorname{GLD}(\alpha, \beta)$, of a random variable $\mathrm{X}$ has probability density function (pdf) given by

$h(x, \alpha, \beta)=\alpha \beta e^{-\beta x}\left(1+e^{-\beta x}\right)^{-(\alpha+1)}, \quad-\infty<x<\infty$

and the cumulative distribution function $(\mathrm{CDF})$ given by

$H(x, \alpha, \beta)=\left(1+e^{-\beta x}\right)^{-\alpha}, \quad-\infty<x<\infty$

Where $\beta>0$ is the scale parameter and $\alpha>0$ is the shape parameter. Olapade (2000) obtained some properties for Type $\operatorname{I~} \operatorname{GLD}(\alpha, \beta)$. The pdf (1.2) has been obtained by compounding of extreme value distribution with a gamma distribution. It is observed by Balakrishnan and Leung (1988) that this distribution is skewed and kurtosis coefficient is greater than the standard logistic distribution.

In this paper, we are using Type $\operatorname{I~} \operatorname{GLD}(\alpha, \beta)$ given by Balakrishnan and Leung (1988), to study the Skew Type $\operatorname{I} \operatorname{GLD}(\alpha, \beta)$, with skeweness parameter $\lambda \in \mathrm{R}$, and will be denoted as $\operatorname{S}^{\prime} \operatorname{GLD}(\alpha, \beta, \lambda)$. The pdf and $\operatorname{CDF}$ of $\operatorname{S} \operatorname{GLD}(\alpha, \beta, \lambda)$ are obtained in explicit forms. Here, we derive the general statistical properties of the $\operatorname{S} \operatorname{GLD}(\alpha, \beta, \lambda)$ such as: moment generating function (mgf), characteristic function (ch. f), Laplace and Fourier 
transformation, expressions for nth moment including the first four moments, skewness and kurtosis coefficients, mean deviation about the mean and median are obtained. Rényi entropy and order statistic for $\operatorname{S}^{\prime} \operatorname{GLD}(\alpha, \beta, \lambda)$ are also given. Moreover, we consider the general case by inclusion of location and scale parameters. Graphical illustration of pdf, CDF of $S^{\prime} G L D(\alpha, \beta, \lambda)$, skewness and kurtosis have been represented. It should be noted that some known results of Nadarajah and Kotz (2007), Nadarajah (2009) are obtained as special cases. The results of the mean deviation, skewness and kurtosis coefficients are given numerically. Finally conclusions of our results are presented.

This paper is organized as follows. In the next section we derive the CDF of $\operatorname{S'GLD}(\alpha, \beta, \lambda)$. In Section 3 we obtained the moment generating function. Skewness and Kurtosis of $\operatorname{S}^{\prime} \operatorname{GLD}(\alpha, \beta, \lambda)$ are given in Section 4 , and in Section 5 , the mean deviation is discussed. Sections 6 and 7 are displayed the Entropy and order statistics. Some important properties are discussed in Section 8. Numerical example is given in Section 9. Finally, Conclusion remarks are provided at Section 10.

\section{2- Cumulative Distribution Function of $\operatorname{S}^{\prime} \operatorname{GLD}(\alpha, \beta, \lambda)$}

In this section, we derive a form of pdf and $\operatorname{CDF}$ of $\operatorname{S} \operatorname{GLD}(\alpha, \beta, \lambda)$, depending on the Type I GLD $(\alpha, \beta)$ given by Balakrishnan and Leung (1988) in (1.2), main feature of the Skew-Symmetric distribution in (1.1) is that a new parameter $\lambda$ is introduced to control skewness and kurtosis.

The pdf of $\operatorname{S}^{\prime} \operatorname{GLD}(\alpha, \beta, \lambda)$ is constructed using formula (1.1), and the pdf $\mathrm{h}($.$) and the$ $\mathrm{CDF} \mathrm{H}($.$) given in (1.2) and (1.3) as follows$

$f(x, \alpha, \beta, \lambda)=2 \alpha \beta e^{-\beta x}\left(1+e^{-\beta x}\right)^{-(\alpha+1)}\left(1+e^{-\beta \lambda x}\right)^{-\alpha},-\infty<x<\infty$

The pdf of $\operatorname{S'GLD}^{\prime}(\alpha, \beta, \lambda)$, given by (2-1) can be expressing in other forms: (a) a single series representation (b) a double series representation, as given in the following lemma.

Lemma (2.1): If $X$ be a random variable having $\operatorname{S} \operatorname{GLD}(\alpha, \beta, \lambda)$, then its pdf is given by (a) $f(x, \alpha, \beta, \lambda)=$

$$
\begin{cases}2 \alpha \beta\left(1+e^{-\beta x}\right)^{-(\alpha+1)} \sum_{j=0}^{\infty}\left(\begin{array}{c}
-\alpha \\
j
\end{array}\right) e^{-\beta x(\lambda j+1)} & x>0 \\
2 \alpha \beta\left(1+e^{\beta x}\right)^{-(\alpha+1)} \sum_{j=0}^{\infty}\left(\begin{array}{c}
-\alpha \\
j
\end{array}\right) e^{\beta x(\alpha+\lambda \alpha+\lambda j)} & x<0\end{cases}
$$

(b) $f(x, \alpha, \beta, \lambda)=$

$$
\begin{cases}2 \alpha \beta \sum_{k=0}^{\infty} \sum_{j=0}^{\infty}\left(\begin{array}{c}
-\alpha \\
j
\end{array}\right)\left(\begin{array}{c}
-\alpha-1 \\
k
\end{array}\right) e^{-\beta x(k+\lambda j+1)} & x>0 \\
2 \alpha \beta \sum_{k=0}^{\infty} \sum_{j=0}^{\infty}\left(\begin{array}{c}
-\alpha \\
j
\end{array}\right)\left(\begin{array}{c}
-\alpha-1 \\
k
\end{array}\right) e^{\beta x(k+\lambda j+\alpha+\lambda \alpha)} & x<0\end{cases}
$$




\section{Proof}

(a) Using binomial expansion for $(1+z)^{-1}$ in the $\operatorname{CDF} H(\lambda x)$ given in (1.2), we get

$$
\begin{aligned}
\mathrm{H}(\lambda x)= & \left(1+e^{-\beta \lambda x}\right)^{-\alpha} \\
& = \begin{cases}\sum_{j=0}^{\infty}\left(\begin{array}{c}
-\alpha \\
j
\end{array}\right) e^{-\beta j \lambda x}, & x>0 \\
e^{\beta \alpha \lambda x} \sum_{j=0}^{\infty}\left(\begin{array}{c}
-\alpha \\
j
\end{array}\right) e^{\beta j \lambda x}, & x<0\end{cases}
\end{aligned}
$$

Substituting from (2.4) into (2.1), the result is obtained.

(b) Also by expanding $\left(1+\mathrm{e}^{-\beta \mathrm{x}}\right)^{-(\alpha+1)}$, the double series representation is given in (2.3).

(a)

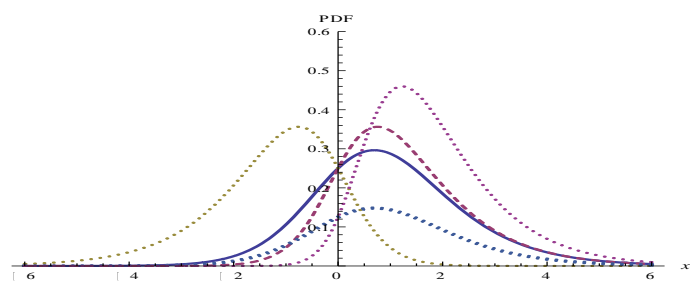

(c)

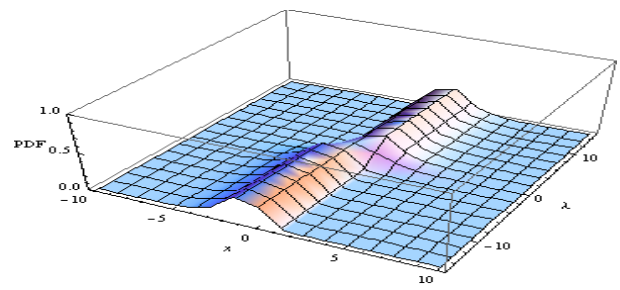

(b)

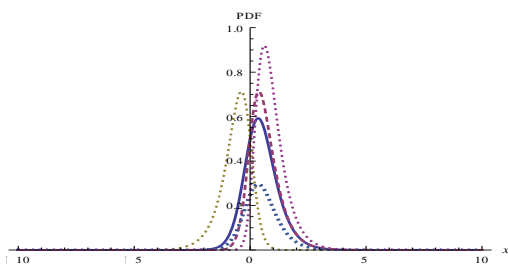

(d)

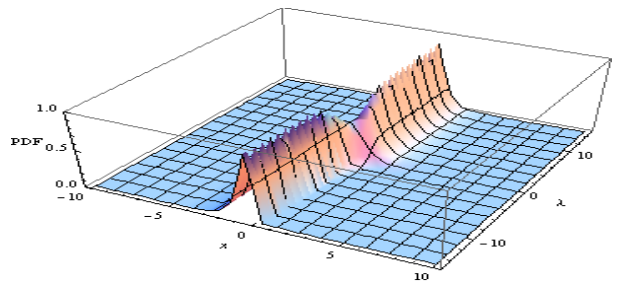

Figure 1: The shapes of pdf of $\operatorname{SGLD}(\alpha, \beta, \lambda)$ (a) when $\beta=1$ with $\alpha=1, \lambda=1$ (Thick line), $\alpha=1, \lambda=2$ (Dashed line), $\alpha=1, \lambda=-2$ (Dotted line), $\alpha=2, \lambda=-2$ (Dashed \& Thick line) and $\alpha=2, \lambda=2$ (Dashed \& Dotted line). (b) when $\beta=2$ with $\alpha$ $=1, \lambda=1$ (Thick line), $\alpha=1, \lambda=2$ (Dashed line), $\alpha=1, \lambda=-2$ (Dotted line), $\alpha=2, \lambda$ $=-2$ (Dashed \& Thick line) and $\alpha=2, \lambda=2$ (Dashed \& Dotted line).(c) when $\alpha=1, \beta$ $=1$ and $\lambda \in(-15,15)$. (d) when $\alpha=1, \beta=2$ and $\lambda \in(-15,15)$.

The calculations through this paper are based on the generalized hypergeometric function $\mathrm{mF}_{\mathrm{n}}$ defined by $m F_{n}\left(\tau_{1}, \tau_{2}, \ldots, \tau_{m} ; \mathcal{V}_{1}, \mathcal{V}_{2}, \ldots, \mathcal{V}_{n} ; x\right)=\sum_{k=0}^{\infty} \frac{\left(\tau_{1}\right)_{k}\left(\tau_{2}\right)_{k} \ldots\left(\tau_{m}\right)_{k} \cdot x^{k}}{\left(\mathcal{V}_{1}\right)_{k}\left(\mathcal{V}_{2}\right)_{k} \ldots\left(\mathcal{V}_{n}\right)_{k} \cdot k !}$ and $(\mathrm{c})_{\mathrm{k}}=\mathrm{c}(\mathrm{c}+1) \ldots \ldots(\mathrm{c}+\mathrm{k}-1)$ denotes the ascending factorial. The properties of this special functions being used can be found in Gradshteyn and Ryzhik (2000) 
Theorem (2.1): If $\mathrm{X}$ is a $\mathrm{S}^{\prime} \mathrm{GLD}(\alpha, \beta, \lambda)$ random variable, then its $\mathrm{CDF}$ is given by

$$
\begin{aligned}
& \text { (a) } F(x, \alpha, \beta, \lambda)= \\
& \begin{cases}2 \alpha \sum_{j=0}^{\infty}\left(\begin{array}{c}
-\alpha \\
j
\end{array}\right)\left\{\frac{A_{1}}{\alpha+\lambda \alpha+\lambda j}+\left[2 \alpha \frac{1}{(\lambda j+1)}\left[A_{2}-A_{3} e^{-\beta x(\lambda j+1)}\right]\right]\right\}, x>0 \\
2 \alpha \sum_{j=0}^{\infty}\left(\begin{array}{c}
-\alpha \\
j
\end{array}\right) A_{4} \frac{e^{\beta x(\alpha+\lambda \alpha+\lambda j)}}{\alpha+\lambda \alpha+\lambda j} & , x<0\end{cases} \\
& \text { (b) } F(x, \alpha, \beta, \lambda)= \\
& \begin{cases}1-\left[2 \alpha \sum_{k=0}^{\infty} \sum_{j=0}^{\infty}\left(\begin{array}{c}
-\alpha \\
j
\end{array}\right)\left(\begin{array}{c}
-\alpha-1 \\
k
\end{array}\right) \frac{e^{-\beta x(k+\lambda j+1)}}{(k+\lambda j+1)}\right] & , x>0 \\
2 \alpha \sum_{k=0}^{\infty} \sum_{j=0}^{\infty}\left(\begin{array}{c}
-\alpha \\
j
\end{array}\right)\left(\begin{array}{c}
-\alpha-1 \\
k
\end{array}\right) \frac{e^{\beta x(k+\lambda j+\alpha+\lambda \alpha)}}{(k+\lambda j+\alpha+\lambda \alpha)} & , x<0\end{cases}
\end{aligned}
$$

Where,

$$
\begin{aligned}
& \mathrm{A}_{1}=2 F_{1}(\alpha+1, \alpha+\lambda \alpha+\lambda j, \alpha+\lambda \alpha+\lambda j+1,-1), \mathrm{A}_{2}=2 F_{1}(\alpha+1, \lambda j+1, \lambda j+2,-1), \\
& \mathrm{A}_{3}=2 F_{1}\left(\alpha+1, \lambda j+1, \lambda j+2,-e^{-\beta x}\right), \quad \text { and } \quad \mathrm{A}_{4}=2 F_{1}(\alpha+1, \alpha+\lambda \alpha+\lambda j, \alpha+\lambda \alpha+ \\
& \left.\lambda j+1,-e^{\beta x}\right)
\end{aligned}
$$

\section{Proof:}

The proof of this theorem has two cases separately, when $\mathrm{X} \geq 0$, andX $<0$, as follows: firstly, when $X \geq 0$ employing the single series form of the pdf given in (2.2), the CDF of $\operatorname{S}^{\prime} \operatorname{GLD}(\alpha, \beta, \lambda)$, can be written as

$$
F(x, \alpha, \beta, \lambda)=P(X \leq x)=\int_{-\infty}^{x} f(x) d x=F(0)+2 \alpha \beta \sum_{j=0}^{\infty}\left(\begin{array}{c}
-\alpha \\
j
\end{array}\right) I_{(x)}, \quad x>0
$$

Where $\mathrm{I}_{(\mathrm{x})}$ is the integral given by

$$
I_{(x)}=\int_{0}^{x} \frac{e^{-\beta x(\lambda j+1)}}{\left(1+e^{-\beta x}\right)^{\alpha+1}} d x
$$

Substituting $\mathrm{y}=\mathrm{e}^{-\beta \mathrm{x}}$, the integral $\mathrm{I}_{(\mathrm{x})}$ reduces to

$=\frac{1}{\beta}\left[\int_{0}^{1} \frac{y^{\lambda j}}{(1+y)^{\alpha+1}} d y-\int_{0}^{e^{-\beta x}} \frac{y^{\lambda j}}{(1+y)^{\alpha+1}} d y\right]=\frac{1}{\beta}\left[I_{1}-I_{2}\right]$

To evaluate the integrals $I_{1}$ and $I_{2}$, we use the formula (3.194.1) in Gradshteyn and Ryzhik (2000), which stated as follows

$\int_{0}^{\mathrm{u}} \frac{\mathrm{x}^{\mu-1}}{(1+\mathrm{bx})^{v}}=\frac{\mathrm{u}^{\mu}}{\mu} 2 \mathrm{~F}_{1}(\mathcal{V}, \mu, 1+\mu,-\mathrm{bu})$

Then, the integrals $I_{1}$ and $I_{2}$ can be calculated as

$$
I_{1}=\frac{A_{2}}{\lambda j+1}, I_{2}=\frac{A_{3} e^{-\beta x(\lambda j+1)}}{\lambda j+1}
$$


Using equations (2.11) and (2.9) we get

$$
I_{(x)}=\frac{1}{\beta(\lambda j+1)}\left[A_{2}-A_{3} e^{-\beta x(\lambda j+1)}\right]
$$

Similarly, $F(0)$ can be calculated as above and we can evaluate it as follows

$F(0)=2 \alpha \sum_{j=0}^{\infty}\left(\begin{array}{c}-\alpha \\ j\end{array}\right) \frac{A_{1}}{\alpha+\lambda \alpha+\lambda j}$

Combining (2.12) and (2.13) into (2.7), the result is yielded.

Second case: when $X<0$, by similar calculation and using (2.10), we get the result. Utilizing the double series representation (2.3), we can also obtain the double series form for the $\operatorname{CDF}$ of $\operatorname{S}^{\prime} \operatorname{GLD}(\alpha, \beta, \lambda)$ given in (2.6). It is clear that the class of the $\operatorname{S} \operatorname{GLD}(\alpha, \beta, \lambda)$ contains the standard logistic distribution (take $\lambda=0, \alpha=1$ and $\beta=1$ ) and the skew logistic distribution (take $\alpha=1$ and $\beta=1$ ). Consequent if we $\operatorname{put}(\alpha=1$ and $\beta=1$ ), the results of Nadarajah and kotz (2007) and Nadarajah (2009) are obtained as special cases. We shall assume that $\lambda \geq 0$, since the corresponding, results for $\lambda<0$ can be obtained using the fact that $-\mathrm{X}$ has the pdf given in (2.1).

(a)

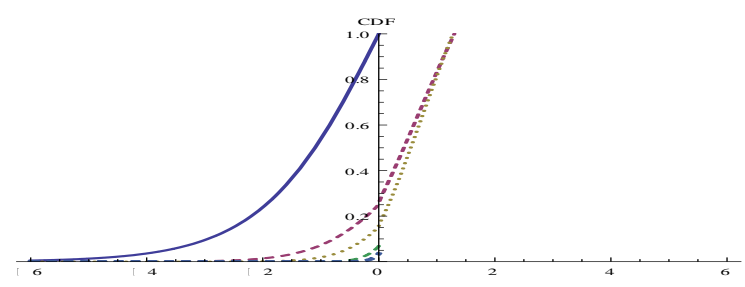

(c)

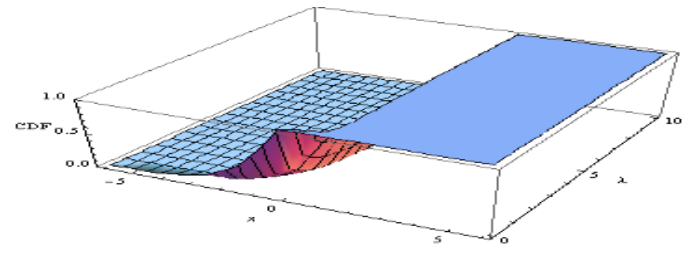

(b)

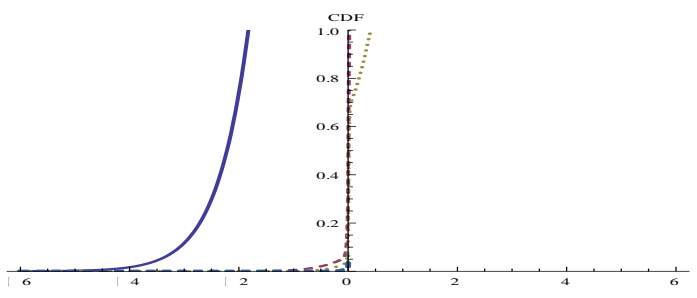

(d)

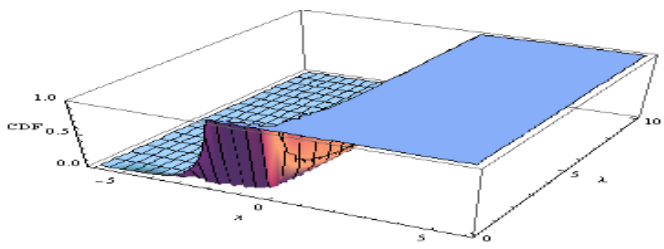

Figure 2: Illustrates the shapes of $\operatorname{CDF}$ of $\operatorname{SGLD}(\alpha, \beta, \lambda)$ (a) when $\lambda=0$ (Thick line), $\lambda=1$ (dashed line), $\lambda=2$ (Dotted line), $\lambda=5$ (Dotted \& Dashed line) and $\lambda=10$ (Dashed \& Dotted line) when $\alpha=1$ and $\beta=1$. (b) when $\lambda=0$ (Thick line), $\lambda=1$ (dashed line), $\lambda=2$ (Dotted line), $\lambda=5$ (Dotted \& Dashed line) and $\lambda=10$ (Dashed \& Dotted line) when $\alpha=2$ and $\beta=1$. (c) when $\alpha=1, \beta=1$ and $\lambda \in(0,10)$. (d) when $\alpha=$ $2, \beta=1$ and $\lambda \in(0,10)$.

\section{3- Moment generating function and characteristic function}

Here, we derive the Moment generating function (mgf) and characteristic function (ch.f) of the random variable $X$ with $\operatorname{S} \operatorname{GLD}(\alpha, \beta, \lambda)$. We consider two forms ofthe pdf, $\mathrm{f}(\mathrm{x}, \alpha, \beta, \lambda)$ when it is taken the one series and double series given by (2.2) and (2.3) respectively. 


\section{Theorem (3.1)}

The mgf of the random variable $X$ with $\operatorname{S} \operatorname{GLD}(\alpha, \beta, \lambda)$ is given by

(a) By using one series representation of pdf given in (2.2)

$$
M_{X}(t)=2 \alpha \sum_{j=0}^{\infty}\left(\begin{array}{c}
-\alpha \\
j
\end{array}\right)\left[\left[\frac{A_{5}}{\alpha+\lambda \alpha+\lambda j+\frac{t}{\beta}}\right]+\left[\frac{A_{6}}{\lambda j+1-\frac{t}{\beta}}\right]\right]
$$

(b) By employing double series representation of pdf given in (2.3)

$$
M_{X}(t)=2 \alpha \beta \sum_{k=0}^{\infty} \sum_{j=0}^{\infty}\left(\begin{array}{c}
-\alpha \\
j
\end{array}\right)\left(\begin{array}{c}
-\alpha-1 \\
k
\end{array}\right)\left[\frac{1}{\beta(k+\lambda j+\lambda \alpha+\alpha)+t}+\frac{1}{\beta(k+\lambda j+1)-t}\right]
$$

Where,

$$
\begin{aligned}
& \mathrm{A}_{5}=2 F_{1}\left(\alpha+1, \alpha+\lambda \alpha+\lambda j+\frac{t}{\beta}, \alpha+\lambda \alpha+\lambda j+\frac{t}{\beta}+1,-1\right), \quad \text { and } \mathrm{A}_{6}=2 F_{1}(\alpha+1, \lambda j+ \\
& \left.1-\frac{t}{\beta}, \lambda j+2-\frac{t}{\beta},-1\right)
\end{aligned}
$$

\section{Proof:}

(a) let $f(x, \alpha, \beta, \lambda)$ given in (2.2), then

$M_{X}(t)=\mathrm{E}\left(e^{t X}\right)=\int_{-\infty}^{\infty} e^{t x} f(x, \alpha, \beta, \lambda) d x=2 \alpha \beta \sum_{j=0}^{\infty}\left(\begin{array}{c}-\alpha \\ j\end{array}\right)\left[I_{3}+I_{4}\right]$

Where, $I_{3}=\int_{-\infty}^{0} \frac{e^{\beta x\left(\alpha+\lambda \alpha+\lambda j+\frac{t}{\beta}\right)}}{\left(1+e^{\beta x}\right)^{\alpha+1}} d x$, and $I_{4}=\int_{0}^{\infty} \frac{e^{-\beta x\left(\lambda j+1-\frac{t}{\beta}\right)}}{\left(1+e^{-\beta x}\right)^{\alpha+1}} d x$

substituting $\mathrm{y}=\mathrm{e}^{\beta \mathrm{x}}$, the integral $\mathrm{I}_{3}$ reduces to

$$
I_{3}=\frac{1}{\beta} \int_{0}^{1} \frac{y^{\alpha+\lambda \alpha+\lambda j+\frac{t}{\beta}-1}}{(1+y)^{\alpha+1}} d y
$$

and the integral $\mathrm{I}_{4}$ take the following form by using $\mathrm{y}=\mathrm{e}^{-\beta \mathrm{x}}$

$I_{4}=\frac{1}{\beta} \int_{0}^{1} \frac{y^{\lambda j-\frac{t}{\beta}}}{(1+y)^{\alpha+1}} d y$

using the formula given in (2.10), the integrals $\mathrm{I}_{3}$ and $\mathrm{I}_{4}$ can be calculated as

$$
I_{3}=\frac{1}{\beta}\left[\frac{A_{5}}{\alpha+\lambda \alpha+\lambda j+\frac{t}{\beta}}\right] \text { and } \quad I_{4}=\frac{1}{\beta}\left[\frac{A_{6}}{\lambda j+1-\frac{t}{\beta}}\right]
$$

From equations (3.1) and (3.4) we get the result (a).

(b) The double series representation of pdf given in (2.3) give

$$
M_{X}(t)=2 \alpha \beta \sum_{k=0}^{\infty} \sum_{j=0}^{\infty}\left(\begin{array}{c}
-\alpha \\
j
\end{array}\right)\left(\begin{array}{c}
-\alpha-1 \\
k
\end{array}\right)\left[I_{5}+I_{6}\right]
$$

where, $I_{5}=\int_{-\infty}^{0} e^{\beta x\left(k+\lambda j+\alpha+\lambda \alpha+\frac{t}{\beta}\right)} d x, \quad$ and, $\quad I_{6}=\int_{0}^{\infty} e^{-\beta x\left(k+\lambda j+1-\frac{t}{\beta}\right)} d x$

In the same manner, we can obtain the result (b), which completes the proof. 


\section{Remark (3.1)}

The characteristic function of the $\operatorname{S} \operatorname{GLD}(\alpha, \beta, \lambda)$ random variable is given by (a) one series representation of pdf given in (2.2)

$\emptyset_{x}(t)=2 \alpha \sum_{j=0}^{\infty}\left(\begin{array}{c}-\alpha \\ j\end{array}\right)\left[\left[\frac{A_{7}}{\alpha+\lambda \alpha+\lambda j+\frac{i t}{\beta}}\right]+\left[\frac{A_{8}}{\lambda j+1-\frac{i t}{\beta}}\right]\right]$

(b) double series representation of pdf given in (2.3)

$$
\emptyset_{x}(t)=2 \alpha \beta \sum_{k=0}^{\infty} \sum_{j=0}^{\infty}\left(\begin{array}{c}
-\alpha \\
j
\end{array}\right)\left(\begin{array}{c}
-\alpha-1 \\
k
\end{array}\right)\left[\frac{1}{\beta(k+\lambda j+\lambda \alpha+\alpha)+i t}+\frac{1}{\beta(k+\lambda j+1)-i t}\right]
$$

Where,

$\mathrm{A}_{7}=2 F_{1}\left(\alpha+1, \alpha+\lambda \alpha+\lambda j+\frac{i t}{\beta}, \alpha+\lambda \alpha+\lambda j+\frac{i t}{\beta}+1,-1\right), \quad \mathrm{A}_{8}=2 F_{1}(\alpha+1, \lambda j+1-$ $\left.\frac{i t}{\beta}, \lambda j+2-\frac{i t}{\beta},-1\right)$,

and $\mathrm{i}=\sqrt{-1}$ is the complex imaginary unit.

The resulting of Nadarajah and kotz (2007) and Nadarajah (2009) for the $m g f \mathrm{M}_{\mathrm{X}}(\mathrm{t})$, and ch.f. $\emptyset_{\mathrm{X}}(\mathrm{t})$ are obtained as special cases by taking $(\alpha=1$ and $\beta=1)$ in (3.1) and (3.5).

Other useful properties of $\operatorname{S}^{\prime} \operatorname{GLD}(\alpha, \beta, \lambda)$ are the Laplace and Fourier Transforms, which are given by the following remarks (3.2) and (3.3), for the two pdf given in (2.2) and (2.3).

\section{Remark (3.2)}

The Laplace Transform of the random variable $X$ having $\operatorname{S'GLD}(\alpha, \beta, \lambda)$ is:

(a) $L \quad(t)=E\left(e^{-t x}\right)=2 \alpha \sum_{j=0}^{\infty}\left(\begin{array}{c}-\alpha \\ j\end{array}\right)\left[\left[\frac{A_{9}}{\alpha+\lambda \alpha+\lambda j-\frac{t}{\beta}}\right]+\left[\frac{A_{10}}{\lambda j+1+\frac{t}{\beta}}\right]\right]$

(b) $L(t)=2 \alpha \beta \sum_{k=0}^{\infty} \sum_{j=0}^{\infty}\left(\begin{array}{c}-\alpha \\ j\end{array}\right)\left(\begin{array}{c}-\alpha-1 \\ k\end{array}\right)\left[\frac{1}{\beta(k+\lambda j+\lambda \alpha+\alpha)-t}+\frac{1}{\beta(k+\lambda j+1)+t}\right]$

Where,

$\mathrm{A}_{9}=2 F_{1}\left(\alpha+1, \alpha+\lambda \alpha+\lambda j-\frac{t}{\beta}, \alpha+\lambda \alpha+\lambda j-\frac{t}{\beta}+1,-1\right), \quad$ and $\quad \mathrm{A}_{10}=2 F_{1}(\alpha+1, \lambda j+$ $\left.1+\frac{t}{\beta}, \lambda j+2+\frac{t}{\beta},-1\right)$

\section{Remark (3.3)}

The Fourier Transform of the random variable $X$ having $\operatorname{S} \operatorname{GLD}(\alpha, \beta, \lambda)$ is:

(a) $F o(t)=E\left(e^{-i t x}\right)=2 \alpha \sum_{j=0}^{\infty}\left(\begin{array}{c}-\alpha \\ j\end{array}\right)\left[\left[\frac{A_{11}}{\alpha+\lambda \alpha+\lambda j-\frac{i t}{\beta}}\right]+\left[\frac{A_{12}}{\lambda j+1+\frac{i t}{\beta}}\right]\right]$

(b) $F o(t)=2 \alpha \beta \sum_{k=0}^{\infty} \sum_{j=0}^{\infty}\left(\begin{array}{c}-\alpha \\ j\end{array}\right)\left(\begin{array}{c}-\alpha-1 \\ k\end{array}\right)\left[\frac{1}{\beta(k+\lambda j+\lambda \alpha+\alpha)-i t}+\frac{1}{\beta(k+\lambda j+1)+i t}\right]$ 
Where,

$$
\begin{aligned}
& \mathrm{A}_{11}=2 F_{1}\left(\alpha+1, \alpha+\lambda \alpha+\lambda j-\frac{i t}{\beta}, \alpha+\lambda \alpha+\lambda j-\frac{i t}{\beta}+1,-1\right), \text { and } \mathrm{A}_{12}=2 F_{1}(\alpha+1, \lambda j+ \\
& \left.1+\frac{i t}{\beta}, \lambda j+2+\frac{i t}{\beta},-1\right) .
\end{aligned}
$$

\section{4- Skewness and kurtosis coefficients}

The skewness coefficient is measured by $\gamma_{1}=\frac{M_{3}}{\sigma^{3}}$, and the kurtosis coefficient is measured by $\gamma_{2}=\frac{M_{4}}{\sigma^{4}}$, where $M_{3}$ and $M_{4}$ are the third and fourth moments about the mean and, $\sigma^{2}$ is the variance of the random variable $X$ having $\operatorname{S} \operatorname{GLD}(\alpha, \beta, \lambda)$.

We firstly derive the $n^{\text {th }}$ moment of the random variable $X$ having pdf given in (2.3) by the following theorem.

\section{Theorem (4.1)}

The $\mathrm{n}^{\mathrm{th}}$ moment of the $\operatorname{S}^{\prime} \operatorname{GLD}(\alpha, \beta, \lambda)$ random variable $\mathrm{X}$ is

(a) If $\mathrm{n}$ is even order

$$
\mathrm{E}\left(X^{n}\right)=\frac{4 \alpha n !}{\beta^{n}} \sum_{k=0}^{\infty} \sum_{j=0}^{\infty}\left(\begin{array}{c}
-\alpha \\
j
\end{array}\right)\left(\begin{array}{c}
-\alpha-1 \\
k
\end{array}\right) \cdot \frac{1}{(k+\lambda j+1)^{n+1}}
$$

(b) If $\mathrm{n}$ is odd

$$
\mathrm{E}\left(X^{n}\right)=\frac{2 \alpha n !}{\beta^{n}} \sum_{k=0}^{\infty} \sum_{j=0}^{\infty}\left(\begin{array}{c}
-\alpha \\
j
\end{array}\right)\left(\begin{array}{c}
-\alpha-1 \\
k
\end{array}\right) \cdot\left[\frac{1}{(k+\lambda j+1)^{n+1}}+\frac{(-1)^{\mathrm{n}}}{(k+\alpha+\lambda j+\lambda \alpha)^{n+1}}\right]
$$

\section{Proof:}

$$
\mathrm{E}\left(X^{n}\right)=\int_{-\infty}^{\infty} x^{n} f(x, \alpha, \beta, \lambda) d x
$$

(a) If $\mathrm{n}$ is even

$$
\mathrm{E}\left(X^{n}\right)=2 \int_{0}^{\infty} x^{n} f(x, \alpha, \beta, \lambda) d x
$$

using (2.3), we get

$$
\mathrm{E}\left(X^{n}\right)=4 \alpha \beta \sum_{k=0}^{\infty} \sum_{j=0}^{\infty}\left(\begin{array}{c}
-\alpha \\
j
\end{array}\right)\left(\begin{array}{c}
-\alpha-1 \\
k
\end{array}\right) \cdot \frac{\Gamma(n+1)}{\beta^{n+1}(k+\lambda j+1)^{n+1}}
$$

Applying the properties of gamma function the result is obtained.

(b) If $\mathrm{n}$ is odd order

$$
\mathrm{E}\left(X^{n}\right)=\int_{-\infty}^{0} x^{n} f(x, \alpha, \beta, \lambda) d x+\int_{0}^{\infty} x^{n} f(x, \alpha, \beta, \lambda) d x
$$


Applying (2.3), and the properties of gamma function we get the result. Consequently, the first four moments of $\mathrm{X}$ can be obtained as

$$
\begin{aligned}
& \mathrm{E}(X)=\frac{2 \alpha}{\beta} \sum_{k=0}^{\infty} \sum_{j=0}^{\infty}\left(\begin{array}{c}
-\alpha \\
j
\end{array}\right)\left(\begin{array}{c}
-\alpha-1 \\
k
\end{array}\right) \cdot\left[\frac{1}{(k+\lambda j+1)^{2}}-\frac{1}{(k+\alpha+\lambda j+\lambda \alpha)^{2}}\right] \\
& \mathrm{E}\left(X^{2}\right)=\frac{8 \alpha}{\beta^{2}} \sum_{k=0}^{\infty} \sum_{j=0}^{\infty}\left(\begin{array}{c}
-\alpha \\
j
\end{array}\right)\left(\begin{array}{c}
-\alpha-1 \\
k
\end{array}\right) \cdot \frac{1}{(k+\lambda j+1)^{3}} \\
& \mathrm{E}\left(X^{3}\right)=\frac{12 \alpha}{\beta^{3}} \sum_{k=0}^{\infty} \sum_{j=0}^{\infty}\left(\begin{array}{c}
-\alpha \\
j
\end{array}\right)\left(\begin{array}{c}
-\alpha-1 \\
k
\end{array}\right) \cdot\left[\frac{1}{(k+\lambda j+1)^{4}}-\frac{1}{(k+\alpha+\lambda j+\lambda \alpha)^{4}}\right] \\
& \mathrm{E}\left(X^{4}\right)=\frac{96 \alpha}{\beta^{4}} \sum_{k=0}^{\infty} \sum_{j=0}^{\infty}\left(\begin{array}{c}
-\alpha \\
j
\end{array}\right)\left(\begin{array}{c}
-\alpha-1 \\
k
\end{array}\right) \cdot \frac{1}{(k+\lambda j+1)^{5}}
\end{aligned}
$$

(a)

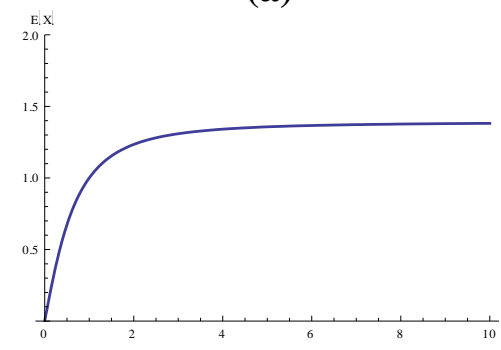

(c)

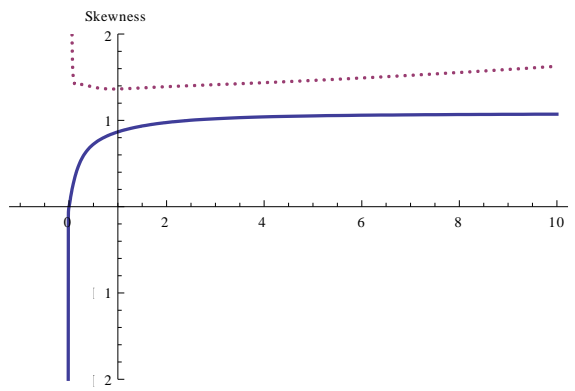

(b)

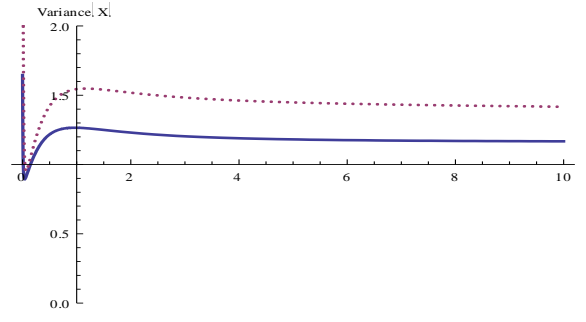

(d)



Figure 2: Illustrates the shapes of (a) the expectation value $\operatorname{E}(X)$ of $\operatorname{S} \operatorname{GLD}(\alpha, \beta, \lambda)$, when $\lambda \epsilon(0,10), \beta=1$ and $\alpha=2$ (Thick line) and $\alpha=2$ (dashed line). (b) the variance $\operatorname{var}(\mathrm{X})$, when $\lambda \in(0,10), \beta=2$ and $\alpha=1$ (Thick line) and $\alpha=1.5$ (dashed line). (c) the skewness coefficient, when $\lambda \epsilon(-1,10), \beta=1$ and $\alpha=1$ (Thick line) and $\alpha=2$ (dashed line). (d) the kurtosis coefficient, when $\lambda \in(-1,10), \beta=1$ and $\alpha=1$ (Thick line) and $\alpha$ $=1.5$ (dashed line).

Therefore, using this four moments, we can easily obtain the variance $\sigma^{2}$, the skewness coefficient $\gamma_{1}$ and kurtosis coefficient $\gamma_{2}$, which will be illustrated here numerically in section (9). If ( $\alpha=1$ and $\beta=1$ ) the results of Nadarajah and kotz (2007) and Nadarajah (2009) are obtained as special cases. 


\section{5- Mean deviation}

The amount of scatter in a population is evidently measured to some extent by the totality of deviation from the mean and median. These are known as the mean deviation about the mean, $\mu$ and the mean deviation about the median, $M$ defined by

$\delta_{1}(X)=\int_{-\infty}^{\infty}|x-\mu| f(x, \alpha, \beta, \lambda) d x$ and,

$\delta_{2}(X)=\int_{-\infty}^{\infty}|x-M| f(x, \alpha, \beta, \lambda) d x$

Respectively, where $\mu=E(X)=$ expectation of $\operatorname{S}^{\prime} \operatorname{GLD}(\alpha, \beta, \lambda)$ random variable $X$.

\section{Theorem (5.1):}

The mean deviation about the mean $\mu$ of a random variable $X$ having $\operatorname{S} \operatorname{GLD}(\alpha, \beta, \lambda)$ is giving as:

$\delta_{1}(X)= \begin{cases}\delta_{11}(\mu), & \text { if } \mu \leq 0 \\ \delta_{12}(\mu), & \text { if } \mu \geq 0\end{cases}$

Where,

$$
\begin{aligned}
& \delta_{11}(\mu)=2 \alpha \beta \sum_{k=0}^{\infty} \sum_{j=0}^{\infty}\left(\begin{array}{c}
-\alpha \\
j
\end{array}\right)\left(\begin{array}{c}
-\alpha-1 \\
k
\end{array}\right)\left[B_{1}{ }^{2}\left(2 e^{\beta \mu(k+\lambda j+\lambda \alpha+\alpha)}-1\right)+B_{2}{ }^{2}\right]-\mu, \\
& \delta_{12}(\mu)=2 \alpha \beta \sum_{k=0}^{\infty} \sum_{j=0}^{\infty}\left(\begin{array}{c}
-\alpha \\
j
\end{array}\right)\left(\begin{array}{c}
-\alpha-1 \\
k
\end{array}\right)\left[B_{2}{ }^{2}\left(2 e^{\beta \mu(k+\lambda j+1)}-1\right)+B_{1}{ }^{2}\right]+\mu, \\
& B_{1}=\frac{1}{\beta(k+\lambda j+\lambda \alpha+\alpha)} \quad, \quad \text { and, } \quad B_{2}=\frac{1}{\beta(k+\lambda j+1)}
\end{aligned}
$$

\section{Proof:}

$\delta_{1}(\mathrm{X})$ can be written in following form given by Nadarajah (2009) as:

$$
\delta_{1}(X)=2 \mu F(\mu)-\mu-\int_{-\infty}^{\mu} x f(x, \alpha, \beta, \lambda) d x+\int_{\mu}^{\infty} x f(x, \alpha, \beta, \lambda) d x(5.1)
$$

depending on two cases, when $\mu \leq 0$ and $\mu \geq 0$, and the calculations are using the double series form given in (2.3). When $\mu \leq 0$ we have from (2.6)

$F(\mu)=2 \alpha \sum_{k=0}^{\infty} \sum_{j=0}^{\infty}\left(\begin{array}{c}-\alpha \\ j\end{array}\right)\left(\begin{array}{c}-\alpha-1 \\ k\end{array}\right) \beta B_{1} e^{\beta \mu(k+\lambda j+\lambda \alpha+\alpha)}$

The integrals in (5.1) can be obtained by elementary calculation as following

$\int_{-\infty}^{\mu} x f(x, \alpha, \beta, \lambda) d x=$

$2 \alpha \beta \sum_{k=0}^{\infty} \sum_{j=0}^{\infty}\left(\begin{array}{c}-\alpha \\ j\end{array}\right)\left(\begin{array}{c}-\alpha-1 \\ k\end{array}\right)\left[B_{1}\left(\mu-B_{1}\right) e^{\beta \mu(k+\lambda j+\lambda \alpha+\alpha)}\right]$

and

$\int_{\mu}^{\infty} x f(x, \alpha, \beta, \lambda) d x=2 \alpha \beta \sum_{k=0}^{\infty} \sum_{j=0}^{\infty}\left(\begin{array}{c}-\alpha \\ j\end{array}\right)\left(\begin{array}{c}-\alpha-1 \\ k\end{array}\right)\left[B_{1}\left(B_{1}-\mu\right) e^{\beta \mu(k+\lambda j+\lambda \alpha+\alpha)}+\right.$

$\left.B_{2}^{2}-B_{1}^{2}\right]$

Substituting from (5.2), (5.3) and (5.4) in (5.1) the result of $\delta_{11}(\mu)$ is obtained. 
Now, when $\mu \geq 0$, we have

$F(\mu)=1-2 \alpha \sum_{k=0}^{\infty} \sum_{j=0}^{\infty}\left(\begin{array}{c}-\alpha \\ j\end{array}\right)\left(\begin{array}{c}-\alpha-1 \\ k\end{array}\right) \frac{e^{-\beta \mu(k+\lambda j+1)}}{(k+\lambda j+1)}$

The integrals in (5.1) are evaluating as:

$\int_{-\infty}^{\mu} x f(x, \alpha, \beta, \lambda) d x=2 \alpha \beta \sum_{k=0}^{\infty} \sum_{j=0}^{\infty}\left(\begin{array}{c}-\alpha \\ j\end{array}\right)\left(\begin{array}{c}-\alpha-1 \\ k\end{array}\right)\left[B_{2}{ }^{2}+B_{1}{ }^{2}-B_{2}(\mu+\right.$

$\left.\left.B_{2}\right) e^{-\beta \mu(k+\lambda j+1)}\right]$

and

$\int_{\mu}^{\infty} x f(x, \alpha, \beta, \lambda) d x=$

$2 \alpha \beta \sum_{k=0}^{\infty} \sum_{j=0}^{\infty}\left(\begin{array}{c}-\alpha \\ j\end{array}\right)\left(\begin{array}{c}-\alpha-1 \\ k\end{array}\right)\left[B_{2}\left(\mu+B_{2}\right)\left(e^{-\beta \mu(k+\lambda j+1)}\right)\right]$

employing (5.5),(5.6) and (5.7) in (5.1) the result of $\delta_{12}(\mu)$ yields.

In the same manner, we can get the mean deviation about the median $\delta_{2}(\mathrm{X})$ by replacing M by $\mu$. We note that.If $(\alpha=1$ and $\beta=1)$ in $\delta_{11}(\mu)$ and $\delta_{12}(\mu)$, the results of Nadarajah (2009) are obtained as special cases.

\section{6- Entropy}

The concept of entropy has been successfully applied in a variety of fields including statistical mechanics, statistic, stock market analysis, queuing theory, image analysis and reliability estimation. (see, e. g., Kapur (1993)). The entropy is a measure the variation of the uncertainty associated with distribution of a random variable X. Notice however that entropy is no longer positive, in fact it can become arbitrary large negative. The Renyi entropy is defined by

$J_{R}(\gamma)=\frac{1}{1-\gamma} \log \left[\int_{-\infty}^{\infty} f^{\gamma}(x, \alpha, \beta, \lambda) d x\right]$

Where $\gamma>0$, and $\gamma \neq 1$ (Renyi 1961). Renyi's entropies are more flexible than Shannon entropies and include Shannon entropies as special case.

\section{Theorem (6.1):}

If $\mathrm{X}$ is a random variable having $\operatorname{S} \operatorname{GLD}(\alpha, \beta, \lambda)$ with pdf given in (2.2). The Renyi entropy is

$\mathrm{J}_{R}(\gamma)=\log \left(\frac{1}{2 \alpha \beta}\right)+\frac{1}{1-\gamma}\left\{2 \alpha \sum_{j=0}^{\infty}\left(\begin{array}{c}-\gamma \alpha \\ j\end{array}\right)\left[\frac{C_{1}}{\gamma+\lambda j}+\frac{C_{2}}{\lambda j+\gamma \alpha+\lambda \gamma \alpha}\right]\right\}$

Where,

$C_{1}=2 F_{1}(\gamma(\alpha+1), \gamma+\lambda j, \gamma+\lambda j+1,-1), \quad$ and $\quad C_{2}=2 F_{1}(\gamma(\alpha+1), \lambda j+\gamma \alpha+$ $\lambda \gamma \alpha, \lambda j+\gamma \alpha+\lambda \gamma \alpha+1,-1)$

Proof: Using the single series representation of $\mathrm{f}(\mathrm{x}, \alpha, \beta, \lambda)$ given in (2.2), we get

$\int_{-\infty}^{\infty} f^{\gamma}(x, \alpha, \beta, \lambda) d x=(2 \alpha \beta)^{\gamma} e^{-\beta x} \sum_{j=0}^{\infty}\left(\begin{array}{c}-\gamma \alpha \\ j\end{array}\right)\left[\mathrm{I}_{7}+\mathrm{I}_{8}\right]$ 
Where

$$
I_{7}=\int_{0}^{\infty} \frac{e^{-\beta x(\gamma+\lambda j)}}{\left(1+e^{-\beta x}\right)^{\gamma(\alpha+1)}} d x, \quad \text { and }, \quad I_{8}=\int_{-\infty}^{0} \frac{e^{\beta x(\lambda j+\gamma \alpha+\lambda \gamma \alpha)}}{\left(1+e^{\beta x}\right)^{\gamma(\alpha+1)}} d x
$$

The integrals $\mathrm{I}_{7}$ and $\mathrm{I}_{8}$ can be calculated as above in (2.10)

$$
\mathrm{I}_{7}=\frac{C_{1}}{\beta(\gamma+\lambda j)} \text {, and } \mathrm{I}_{8}=\frac{C_{2}}{\beta(\lambda j+\gamma \alpha+\lambda \gamma \alpha)}
$$

Substituting from (6.3) in (6.2) we get

$$
\int_{-\infty}^{\infty} f^{\gamma}(x, \alpha, \beta, \lambda) d x=\frac{(2 \alpha \beta)^{\gamma}}{\beta} \sum_{j=0}^{\infty}\left(\begin{array}{c}
-\gamma \alpha \\
j
\end{array}\right)\left[\frac{C_{1}}{\gamma+\lambda j}+\frac{C_{2}}{\lambda j+\gamma \alpha+\lambda \gamma \alpha}\right]
$$

Therefore, using (6.1) and (6.4) the result is obtained.

\section{7- Order Statistics}

Suppose $\mathrm{X}_{1}, \mathrm{X}_{2}, \ldots \mathrm{X}_{\mathrm{n}}$ is a random sample of size $\mathrm{n}$ from a population with pdf $f(\mathrm{x}, \alpha, \beta, \lambda)$ Let $\mathrm{X}_{1: \mathrm{n}}<\mathrm{X}_{2: \mathrm{n}}<\cdots<\mathrm{X}_{\mathrm{n}: \mathrm{n}}$ denote the corresponding order statistics it is well known that the pdf and CDF of the $\mathrm{k}^{\text {th }}$ order statistics say $\mathrm{Y}_{\mathrm{k}}=\mathrm{X}_{\mathrm{k}: \mathrm{n}}$ are given respectively by

$$
\begin{aligned}
& f_{k}\left(y_{k}\right)=\frac{n !}{(k-1) !(n-k) !}\left[F\left(y_{k}\right)\right]^{k-1}\left[1-F\left(y_{k}\right)\right]^{n-k} f\left(y_{k}\right), \text { and } \\
& F_{k}\left(y_{k}\right)=\sum_{r=k}^{n}\left(\begin{array}{l}
n \\
r
\end{array}\right)\left[F\left(y_{k}\right)\right]^{r}\left[1-F\left(y_{k}\right)\right]^{n-r} \quad, \quad k=1,2, \ldots, n
\end{aligned}
$$

If the population $X$ having $\operatorname{S}^{\prime} \operatorname{GLD}(\alpha, \beta, \lambda)$ with pdf (2.1). The pdf of the $\mathrm{k}^{\text {th }}$ order statistic $\mathrm{Y}_{\mathrm{k}}=\mathrm{X}_{\mathrm{k}: \mathrm{n}}$ is given as

$f_{k}\left(y_{k}, \alpha, \beta, \lambda\right)=\frac{4 \alpha^{2} \beta n !}{(k-1) !(n-k) !} \sum_{u=0}^{\infty}(-1)^{u}\left(\begin{array}{c}n-k \\ u\end{array}\right) e^{-\beta x}\left(1+e^{-\beta x}\right)^{-(\alpha+1)}(1+$
$\left.e^{-\beta \lambda x}\right)^{-\alpha} \sum_{k=0}^{\infty} S^{u+j-1}$

Where, $S=\left[\frac{A_{1}+A_{4}}{\lambda j+\lambda \alpha+\alpha}+\frac{2 \alpha}{\lambda j+1}\left[A_{2}-A_{3} e^{-\beta x(\lambda j+1)}\right]\right]$

The smallest and largest order statistics are of special importance, as are certain functions of order statistics known as the sample median and the range. The sample rang is the difference of the smallest from the largest, $R=Y_{n}-Y_{1}$.Consequently, the pdf of the smallest and largest order statistics are given by

and

$$
\begin{aligned}
f_{1}\left(y_{1}, \alpha, \beta, \lambda\right) & =n f\left(y_{1}, \alpha, \beta, \lambda\right)\left[1-F\left(y_{1}, \alpha, \beta, \lambda\right)\right]^{n-1} \\
& =4 n \alpha^{2} \beta \sum_{u=0}^{\infty}(-1)^{u}\left(\begin{array}{c}
n-k \\
u
\end{array}\right) e^{-\beta x}\left(1+e^{-\beta x}\right)^{-(\alpha+1)}\left(1+e^{-\beta \lambda x}\right)^{-\alpha} S^{u}
\end{aligned}
$$




$$
\begin{aligned}
f_{n}\left(y_{n}, \alpha, \beta, \lambda\right) & =n f\left(y_{n}, \alpha, \beta, \lambda\right)\left[F\left(y_{n}, \alpha, \beta, \lambda\right)\right]^{n-1} \\
& =4 n \alpha^{2} \beta e^{-\beta x}\left(1+e^{-\beta x}\right)^{-(\alpha+1)}\left(1+e^{-\beta \lambda x}\right)^{-\alpha} \sum_{j=0}^{\infty}\left(\begin{array}{c}
-\alpha \\
j
\end{array}\right) S
\end{aligned}
$$

As the same manner, we can obtain the $\mathrm{CDF}$ of the $\mathrm{k}^{\mathrm{th}}$ order statistics $\mathrm{F}_{\mathrm{k}}\left(\mathrm{y}_{\mathrm{k}}, \alpha, \beta, \lambda\right)$.

\section{8- Transformation of variables}

In practice, one often works with the family of distribution generated by linear transformation $Z=\mu+\eta X$, where $X$ has $\operatorname{S} \operatorname{GLD}(\alpha, \beta, \lambda)$. The random variable $Z$ gives the general class of the $\operatorname{S} \operatorname{GLD}(\alpha, \beta, \lambda)$ by inclusion of the location parameter $\mu$ and the scale parameter $\eta$. It is easy to see that the random variable $Z$ having also $\operatorname{S}^{\prime} G L D(\alpha, \beta, \lambda, \mu, \eta)$.

Theorem (8.1): Let $X$ be a random variable having $\operatorname{S}^{\prime} \operatorname{GLD}(\alpha, \beta, \lambda)$, and $Z=\mu+\eta X$. Then the nth moment of the random variable $Z$ is given by $E(Z)^{n}=\sum_{j=0}^{n}\left(\begin{array}{c}n \\ j\end{array}\right) \mu^{n-j} \eta^{j} E\left(X^{j}\right)$.

By elementary calculation, we can prove the theorem. Therefore by illustrating the first four moments of a random variable $X$ given in section (4)
(i) $E(Z)=\mu+\eta E(X)$
(ii) $\operatorname{var}(Z)=\eta^{2} \operatorname{var}(X)$
(iii) $\gamma_{1}(Z)=\eta^{3} \gamma_{1}(X)$
(iv) $\gamma_{2}(Z)=\eta^{4} \gamma_{2}(X)$

\section{9- Numerical Example}

In this section, we express the flexibility of the distribution to account for wide ranges of the skewness and the kurtosis coefficients $\gamma_{1}, \gamma_{2}$ respectively. The mean deviation about the mean is also given for $(\beta=1$ and different values of $\alpha$ and $\lambda)$.

\begin{tabular}{|c|c|c|c|c|c|c|c|c|c|}
\hline$\alpha$ & -2 & -1 & 0 & 1 & 2 & 3 & 4 & 15 & 20 \\
\hline 1 & -- & --- & 0 & 0.86647 & 0.97563 & 1.01933 & 1.04061 & 1.07755 & 1.08035 \\
\hline 2 & --- & --- & 0.00413 & 1.36448 & 1.39063 & 1.41414 & 1.43734 & 1.83258 & 2.06964 \\
\hline 3 & --- & --- & $5.19146 \times 10^{-7}$ & $3.39053 \times 10^{-7}$ & $3.27000 \times 10^{-6}$ & 0.00002 & 0.00005 & 0.00663 & 0.00897812 \\
\hline 4 & --- & --- & $1.45082 \times 10^{-12}$ & $3.51833 \times 10^{-14}$ & $1.15715 \times 10^{-13}$ & $3.90487 \times 10^{-13}$ & $1.20543 \times 10^{-12}$ & $9.39809 \times 10^{-10}$ & $4.33379 \times 10^{-9}$ \\
\hline 15 & --- & --- & $1.97785 \times 10^{-53}$ & $7.24111 \times 10^{-53}$ & $1.58275 \times 10^{-52}$ & $2.77360 \times 10^{-52}$ & $4.29666 \times 10^{-52}$ & $4.29751 \times 10^{-51}$ & $7.38439 \times 10^{-51}$ \\
\hline 20 & --- & --- & $1.09192 \times 10^{-65}$ & $4.07240 \times 10^{-65}$ & $8.95432 \times 10^{-65}$ & $1.57374 \times 10^{-64}$ & $2.44217 \times 10^{-64}$ & $2.45423 \times 10^{-63}$ & $4.21924 \times 10^{-63}$ \\
\hline
\end{tabular}

Table 1: Skewness coefficients of $\operatorname{SGLD}(\alpha, \beta, \lambda)$ for $\beta=1$.

\begin{tabular}{|c|c|c|c|c|c|c|c|c|c|}
\hline$\lambda$ & -2 & -1 & 0 & 1 & 2 & 3 & 4 & 15 & 20 \\
\hline 1 & -- & -- & 2.09904 & 3.46220 & 3.74207 & 3.91780 & 4.01452 & 4.19759 & 4.21212 \\
\hline 2 & -- & -- & 0.000743179 & 5.03561 & 4.90893 & 4.97688 & 5.07502 & 7.00707 & 8.24078 \\
\hline 3 & -- & -- & $1.35007 \times 10^{-9}$ & $7.06498 \times 10^{-9}$ & $1.38168 \times 10^{-7}$ & $1.13417 \times 10^{-6}$ & $5.70119 \times 10^{-6}$ & 0.00350366 & 0.00525244 \\
\hline 4 & -- & -- & $1.87801 \times 10^{-17}$ & $2.99604 \times 10^{-19}$ & $5.63502 \times 10^{-18}$ & $5.01644 \times 10^{-17}$ & $2.80564 \times 10^{-16}$ & $2.39203 \times 10^{-12}$ & $1.83070 \times 10^{-11}$ \\
\hline 15 & -- & -- & $3.65366 \times 10^{-79}$ & $2.50946 \times 10^{-78}$ & $8.05860 \times 10^{-78}$ & $1.86366 \times 10^{-77}$ & $3.58673 \times 10^{-77}$ & $1.12891 \times 10^{-75}$ & $2.54141 \times 10^{-75}$ \\
\hline 20 & -- & -- & $1.32975 \times 10^{-97}$ & $9.46589 \times 10^{-97}$ & $3.07468 \times 10^{-96}$ & $7.15069 \times 10^{-96}$ & $1.38080 \times 10^{-95}$ & $4.38570 \times 10^{-94}$ & $9.88271 \times 10^{-94}$ \\
\hline
\end{tabular}

Table 2: Kurtosis coefficients of $\operatorname{SGLD}(\alpha, \beta, \lambda)$ for $\beta=1$. 


\begin{tabular}{|c|c|c|c|c|c|c|c|c|}
\hline$\lambda$ & -2 & -1 & 0 & 1 & 2 & 3 & 4 & 15 \\
\hline 1 & -- & -- & 2.81142 & $8.11295 \times 10^{41}$ & $5.76309 \times 10^{78}$ & $7.59643 \times 10^{111}$ & $3.91773 \times 10^{143}$ & $1.68200 \times 10^{479}$ \\
\hline 2 & -- & -- & $8.38400 \times 10^{1175}$ & $7.56939 \times 10^{99}$ & $7.16303 \times 10^{159}$ & $1.95846 \times 10^{216}$ & $1.54406 \times 10^{272}$ & $6.71361 \times 10^{928}$ \\
\hline 3 & -- & -- & $2.58245 \times 10^{74879}$ & $3.71719 \times 10^{18946}$ & $2.85677 \times 10^{13656}$ & $6.80111 \times 10^{10758}$ & $2.07035 \times 10^{8982}$ & $2.00770 \times 10^{5932}$ \\
\hline 4 & $\overline{--}$ & -- & $1.62501 \times 10^{15135430}$ & $2.99559 \times 10^{8300509}$ & $1.36044 \times 10^{5807092}$ & $7.21447 \times 10^{4458748}$ & $2.85251 \times 10^{3617515}$ & $4.97957 \times 10^{1200900}$ \\
\hline
\end{tabular}

Table 3: Mean deviation about the mean of $\operatorname{SGLD}(\alpha, \beta, \lambda)$ for $\beta=1$.

\section{0- Conclusion}

From Figure (3), Table (1) and (2), we see that (i) the expectation value $E(X)$ increases as $\lambda$ increases and decreases as $\alpha$ increases. (ii) $\operatorname{var}(\mathrm{x})$ decreases as $|\lambda|$ increases and decreases as $\alpha$ increases.(iii) Skewness coefficient $\gamma_{1}$ increases as $\lambda$ increases and decreases as $\alpha$ increase. (iv) kurtosis coefficients $\gamma_{2}$ initially decreases before increasing as $|\lambda|$ increases and decreases as $\alpha$ increases. (v) from for the standard logistic distribution $\gamma_{1}=0$ and $\gamma_{2}=2$ which means it is symmetric platykurtic (vi) from table 3 , it is clear that the mean deviation about the mean increases when $\lambda$ increases and aincreases. (vii) the flexibility of $\operatorname{SGLD}(\lambda, \alpha, \beta)$ in terms of accommodating more general types of skewness than the ordinary $\operatorname{SDL}(\lambda)$ is illustrated by computing moments and, in particular, skewness and kurtosis coefficients.

\section{1- References}

1. Asgharzadeh, A., Esmaeili, L., Nadarajah, S. and Shih, S.H. (2013). A generalized Skew Logistic Distribution, REVSTAT - Statistical Journal, Volume 11, Number 3, November, 317-338

2. Azzalini, A., (1985). A class of distributions which includes the normal ones. Scandinavian Journal of Statistics, 12, 171-178.

3. Balakrishnan N. and Leung M. Y. (1988). Order Statistics from the Type I Generalized logistic distribution. Communications in Statistics simulation and computation. Vol. 17(1) 25-50.

4. Chakraborty S., Hazarika P.J. and Masoom A.A. (2012). New Skew logistic distribution and its properties. Pak. J. Statist. Vol. 28 (4) 513-524.

5. Gupta A.K., Chong F.C., and Huong W.J. (2002). Some skew-symmetric models. Random oper. Stoch. 133-140.

6. Gupta R.D. and Kunda D. (2010). Generalized Logistic Distribution. J. Appl. Stat. Sci., 12(1) 51-66.

7. Johnson, N.L., Kotz, S., Balakrishnan, N. (1995). Continuous Univariate Distributions, vol. 2Houghton Mifflin co., Boston.

8. Kapur, J.N.: (1993). Maximum Entropy Models in science and Engineering (revised Ed.), Wiley, New York,

9. Lagos-Álvareza B., Jiménez-Gamerob M. D. and Alba-Fernández V. (2011). Bias Correction in the Type I Generalized Logistic Distribution, Communication in Statistics-Simulation and Computation, 40, 511-531. 
10. Nadarajah S. (2009). The skew logistic distribution. ASTA ADV. stat. Anal, 93 187-203.

11. Nadarajah S. and Kotz S. (2007). Skew models I. Acta. App. Math., 981-28.

12. Nadarajah S. and Kotz S. (2006). Skew distribution generated from different families. Acta. App. Math., 911-37.

13. Nassar M.M. and Elmasry A., (2012). A study of Generalized Logistic Distribution. Journal of the Egyptian Mathematical Society, 2012 6-133.

14. Ojo M. O., (2003). A remark on the convolution of the generalized logistic random variables. ASSET serves A, vol.1, No.2.

15. Ojo M. O., (2002). Approximation to the distribution of the sum of the generalized logistic random variables. Kragujevac journal of mathematics. vol.24 135-145.

16. Ojo M. O., (1997). Some relationships between the generalized logistic and other distributions. Statistica, LvII.4 573-579.

17. Olapade A.K. (2005). On negatively Skewed Extended Generalized logistic distribution Kragujevac J. Math. 27 175-182.

18. Olapade A.K., (2004). On extended Type I Generalized logistic distribution. international Journal of Mathematics and Mathematical sciences.

19. Olapade A.K. (2000). Some properties of the Type I Generalized logistic distribution. InterStat. 2

20. Renyi, A. (1961). On measures of entropy and information. In proceedings of the Fourth Berkeley Symposium on Mathematical Statistics and probability. Vol. I.547-561. University of California press. Berkeley.

21. Wahed A.S. and Ali M.M. (2001). The skew-logistic distribution. J. Statist. Res., 35 71-80. 